\title{
Transient Response of Electrons and Phonons in ZnTe Crystals
}

\author{
R. BRAZIS* AND R. RAGUOTIS \\ Semiconductor Physics Institute, A. Goštauto 11, 01108 Vilnius, Lithuania \\ The response of electron and phonon ensemble to the switching on and \\ off electric field $E$ in $n$-type ZnTe crystals is simulated by Monte Carlo \\ method. The results at $T=10 \mathrm{~K}$ show significant accumulation of LO- \\ phonons and the LO phonon band population inversion with respect to the \\ LA band; the inversion is necessary for the stimulated transfer of LO-LA \\ energy difference to photons. The maximum inversion is at $E=7 \mathrm{kV} / \mathrm{cm}$. \\ At $T=300 \mathrm{~K}$ no phonon band inversion but fast (sub-picosecond) drift \\ velocity switching with $\approx 100 \mathrm{GHz}$ repetition is feasible.
}

PACS numbers: 72.10.Di, 72.20.Ht, 72.15.Lh, 78.45.+h

\section{Introduction}

Development of ultrafast optoelectronics challenges for the search of materials exhibiting short times of response to electric field or/and light pulses. One of the main candidate materials is crystalline ZnTe that is widely used in laser optic components. However, its basic characteristics such as the risetimes of electron drift velocity (and mean energy) upon switching on electric field, as well as the times of their decay after switching off the field, are not well known. The riseand decay times are expected to be modified by the electron sub-system interaction with phonons. Monte Carlo (MC) method has been refined recently so as to determine stationary electron- and phonon distributions in electric fields $[1,2]$.

This report presents the study on the rise (decay) time of electron drift velocity and average energy, as well as the maximum phonon number, upon the step-like switching on (off) electric field in $n$-type ZnTe crystals. Special attention is paid to the dynamics of electrons and phonons affected by the second (probing) electric field pulse applied after the initial one with some delay. Optical phonon band population inversion with respect to acoustic one is considered. Results at the lattice temperatures of $T=10 \mathrm{~K}$ and $300 \mathrm{~K}$ are compared.

\section{Input data and results}

ZnTe material parameters were used as listed in Ref. [3]. Electron scattering mechanisms included inelastic processes of polar-optical mode (LO phonon)

*corresponding author; e-mail: brazis@pfi.lt 
emission and absorption, quasi-elastic interaction with longitudinal acoustic (LA) modes, and the elastic process of electron interaction with impurity ions. The last one was accounted for by the third-body exclusion method [4]. LO and LA phonon lifetimes were supposed to be 2 ps and 30 ps.
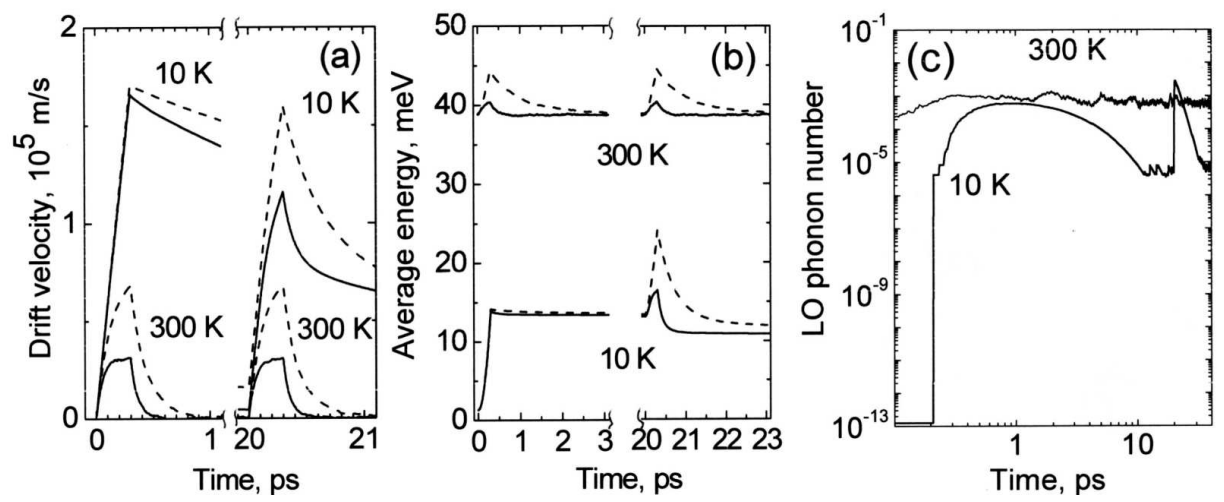

Fig. 1. Electron drift velocity, average energy, and maximum LO-phonon number in ZnTe crystals in response to electric field pulse $(5 \mathrm{kV} / \mathrm{cm}, 300 \mathrm{fs})$ switched on at $t=0$ and repeated at $20 \mathrm{ps}$. Lattice temperature is shown at the curves; $(-)$ with phonon accumulation, (- - ) no accumulation. $N_{\mathrm{LO}}(E)-N_{\mathrm{LO}}(0)$ is plotted for $T=300 \mathrm{~K}$.

At $T=10 \mathrm{~K}$, the drift velocity during the first pulse (300 fs) action grows linearly with time (Fig. 1a) and decays exponentially after the pulse termination. The decay (momentum randomization) time is approximately $5.4 \mathrm{ps}$. If the emitted LO phonons are supposed to disappear immediately (no LO phonon accumulation) then the decay process is slower; the momentum randomization time of $8.6 \mathrm{ps}$ is controlled by electron scattering on LA phonons. Electrons are still moving ballistically when the second (probe) pulse comes after 20 ps delay. The drift velocity growth in time is now sublinear, and its peak value is lower. After the second pulse, momentum randomization process is shorter; it exhibits twoexponent behavior with the time constants of about $0.7 \mathrm{ps}$ and $4.5 \mathrm{ps}$. Due to the non-zero electron drift velocity at the beginning of the second pulse, a remarkable fraction of electrons penetrates deeper in the "active" region of momentum space where their kinetic energy $\hbar^{2} k^{2} / 2 m^{*}$ is higher than the LO phonon energy $\hbar \omega_{\mathrm{LO}}$. Therefore the shorter decaytime is seemingly related to the lifetime of electrons in the active region. At the same $T=10 \mathrm{~K}$, the average electron energy (Fig. 1b) decays slightly in the short time of about 0.2 ps just after the first pulse. This fast process is in relation with the lifetime of electrons in the active region. The average energy remains next for a long time at the level of about one half of the optical phonon energy ( $\hbar \omega_{\mathrm{LO}} \approx 26 \mathrm{meV}$ ). It is a kind of memory effect: electrons have no channel of energy transfer except the quasielastic interaction with LA phonons. The second (probe) electric field pulse forces electrons to access LO-phonon energy 
and lose it by LO-phonon emission. The average energy gets rapidly lower after the second pulse action. The cooling is not complete but it is more remarkable in the case of LO-phonon accumulation than without the accumulation.

At $T=10 \mathrm{~K}$, the most spectacular is the transient response of LO-mode lattice vibrations (Fig. 1c). The growth of the maximum value of phonon distribution function is delayed relative to the front step of the electric field pulse. As the first electrons reach LO-phonon energy, the phonon emission becomes stimulated; it brings about the stepwise increase in LO-phonon number by many orders of magnitude. LO-phonon emission dominates also for nearly 700 fs after switching off the field. After that time the phonon number decreases but it does not return to its thermal-equilibrium value. The second pulse brings about the increase in LO-phonon number over its highest value accessed in the first action.

At $T=300 \mathrm{~K}$, the phonon number being initially quite large $\left[N_{\mathrm{LO}}(0)=\right.$ 0.5914] exhibits only slight increase in electric field. Therefore, the difference $N_{\mathrm{LA}}(E)-N_{\mathrm{LO}}(0)$ is plotted in Fig. 1. LO phonon response shows no step during the first pulse, and rather minor effects of the second pulse. Nevertheless, the very slight increase in phonon number is able to diminish notably the drift velocity and mean energy of electrons. Both the drift velocity and the average energy relaxes fast $(\approx 0.13 \mathrm{ps})$ allowing for high repetition of pulses.
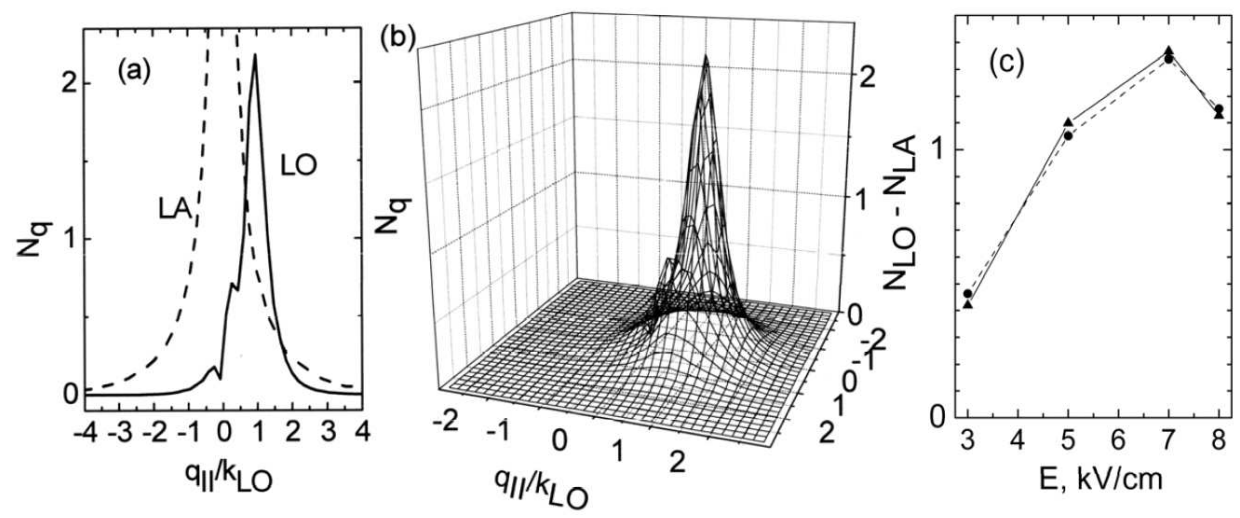

Fig. 2. Phonon distribution in momentum space: (a) in a tube parallel to $\boldsymbol{E}(7 \mathrm{kV} / \mathrm{cm})$ centered at $q_{\perp}=0$; (b) in the $q_{\|} q_{\perp}$ plane; (c) the difference $N_{\mathrm{LO}}-\mathrm{N}_{\mathrm{LA}}$ at the phonon wave vector $q \approx k_{\mathrm{LO}}$. Triangles correspond to the distribution parallel to the field, and dots are for points across the field. $T=10 \mathrm{~K}$.

One can note the possibility of phonon band population inversion at low temperatures (Fig. 2). LO-phonon distribution is centered at the wave vector $q \approx k_{\mathrm{LO}}$ (the wave vector of electrons whose kinetic energy equals to $\hbar \omega_{\mathrm{LO}}$ is $k_{\mathrm{LO}} \approx$ $0.012 \mathrm{q}_{\max } ; q_{\max }=2 \pi / a=1.035 \times 10^{10} \mathrm{~m}^{-1}$, and $a$ is the lattice constant). The inversion of LO-phonon band population with respect to that of the LA phonons is maximal inversion at $E \approx 7 \mathrm{kV} / \mathrm{cm}$. 
In conclusion, the MC study of time response of electron kinetic characteristics on the short switched on and off probe electric field pulses in $n$-type ZnTe crystals shows that sub-picosecond switching of electron drift velocity with the repetition rate of the order of $100 \mathrm{GHz}$ is possible at room temperatures, whereas at low temperatures the processes are much slower due to the LO-phonon accumulation. On the other hand, the low temperature regime manifests significant inversion of LO- and LA-phonon band population that is of interest for the stimulated emission of photons in phonon-difference processes [3].

\section{Acknowledgments}

The work is partially supported by the Lithuanian Agency of International Science and Technology Transfer project 10V-114 in the framework of COST 288 Action.

\section{References}

[1] R. Brazis, R. Raguotis, Acta Phys. Pol. A 107, 339 (2005).

[2] R. Brazis, R. Raguotis, in 8th ICTON Proc. 2, 203 (2006).

[3] R. Brazis, D. Nausewicz, R. Raguotis, Phys. Status Solidi B 244, 1662 (2007).

[4] B.K. Ridley, Quantum Processes in Semiconductors, Clarendon Press, Oxford, 1982. 